\title{
Flexible Duplexing for Maximum Downlink Rate in Multi-tier MIMO Networks
}

\author{
Jacobo Fanjul and Ignacio Santamaria \\ Department of Communications Engineering, University of Cantabria, 39005 Santander, Spain \\ e-mail: \{fanjulj,i.santamaria\}@unican.es
}

\begin{abstract}
In this paper, we propose an algorithm to maximize downlink rate performance in the context of multiple-input multiple-output (MIMO) Heterogeneous Networks (HetNets). Specifically, we evaluate the benefits of flexible duplexing, a promising strategy that consists in combining uplink and downlink cells within the same channel use. In order to handle intercell interference, we rely on the interference alignment (IA) technique, taking into account the impact of the channel estimation errors on the inter-cell interference leakage. Determining the best uplink/downlink configuration is a combinatorial problem, and therefore we consider several approaches to reduce the computational demands of the problem. First, we use a statistical characterization for the average rates achieved by IA in order to avoid the calculation of alignment solutions for all possible settings in the network. Additionally, we propose two hierarchical switching (HS) strategies so that only a subset among the total number of combinations is explored. As a performance baseline, we include in the comparison the conventional time division duplex (TDD) approach and the well-known minimum mean square error (MMSE) decoder. The obtained results show that downlink rates achieved by implementing flexible duplexing and applying inter-cell IA significantly outperform conventional TDD transmissions. Finally, the proposed hierarchical schemes are shown to obtain almost the same rates as exhaustive search with much lower computational cost.
\end{abstract}

Index Terms-Flexible duplexing; heterogeneous networks; interference alignment; statistical analysis; discrete optimization.

\section{INTRODUCTION}

Recently, in the context of the 5-th generation of mobile communications $(5 \mathrm{G})$, heterogeneous networks (HetNets) have arised as a promising set of network topologies comprised of nodes and cells of different characteristics combined within the same system [1]. Among the vast diversity of scenarios under the denomination of HetNets, flexible duplexing (also known as reverse-TDD or dynamic-TDD in the literature) emerges as an access technique that allows both uplink and downlink cells coexisting in the same time and frequency band. This strategy relies on the fact that different uplink/downlink (UL/DL) combinations originate different interference levels at the input of the receivers, hence playing a paramount role in the system performance.

Although the interest in flexible duplexing is quite recent, several studies have been performed in order to analyze the potential benefits that combining uplink and downlink cells can provide. A first general analysis of massive MIMO, small cells and flexible duplexing is discussed in [2], where the first evidence of rate benefits of advanced TDD techniques is included. Further, a reverse-TDD scheme is considered in [3] for massive MIMO HetNets with a dense tier of small cells. Shortly afterwards, an interference management study in the context of flexible duplexing dense HetNets with wireless backhaul is presented in [4]. A more general analysis is performed in [5] building on the so-called $\alpha$-duplex model. Additionally, the coexistence of FDD flexible duplexing systems is studied in [6] in terms of downlink transmit power, and frame structures for interference mitigation in flexible duplexing transmissions can be found in [7], [8], [9].

Besides the UL/DL combination itself, the interference management strategy implemented at the transmitters and receivers in the network has a significant impact on the achieved performance. In this sense, interference alignment (IA) has been shown to provide excellent results in scenarios where the magnitude of the interfering signals is similar to the desired signal level. However, the existing works considering the implementation of IA in HetNets are still scarce. The feasibility of alignment for flexible duplexing 2 -cell networks is analyzed in [10], [11], and authors in [10] establish that the existence of degrees-of-freedom (DoF) benefits due to the implementation of flexible duplexing depends on the network topology. Further DoF evaluation of 2-cell flexible duplexing HetNets is included in [12], whereas these studies are extended to a multi-cell network model in [13]. Additionally, a discussion on the applicability of interference alignment in flexible duplexing experimental deployments can be found in [14].

Nevertheless, despite these research efforts, the task of finding the best UL/DL combination for a given figure of merit (e.g., the total downlink rate in the network) has not been addressed in the existing literature. Therefore, in this work we present an analysis of 2-tier multicell flexible duplexing HetNets where IA precoding and decoding vectors are applied in order to mitigate inter-cell interference. We use statistical expressions to characterize the average rates while reducing the computational cost of exploring the different uplink/downlink combinations. Finally, we propose two hierarchical switching (HS) methods that allow to find the UL/DL combination that maximizes downlink rates with no need of exploring all the possible network configurations, and we evaluate their performance by means of Monte Carlo simulations. 


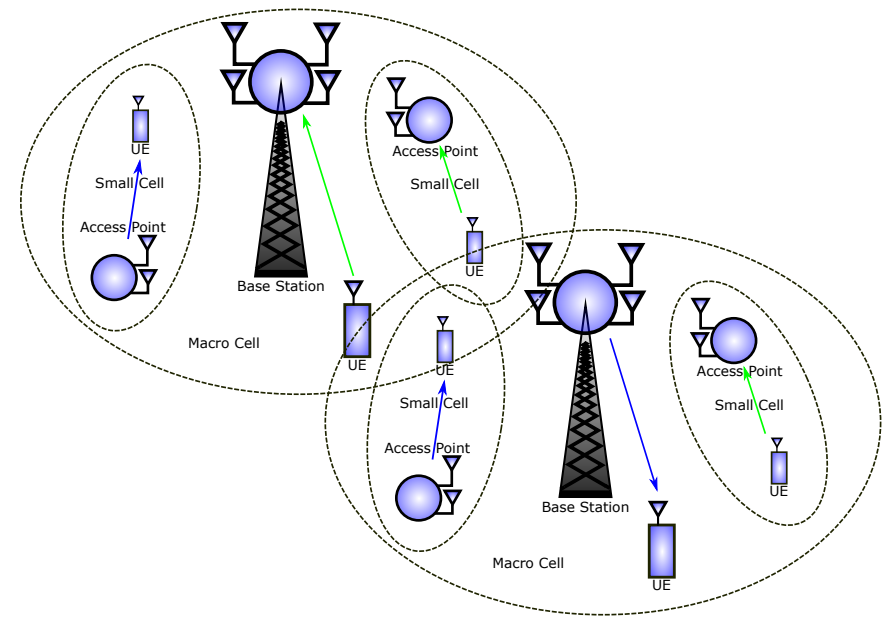

Fig. 1. Example of 2-tier HetNet implementing flexible duplexing.

\section{A. Notation}

Uppercase and lowercase boldface letters will be used for matrices and column vectors, respectively. $(\cdot)^{T}$ will represent transpose, whereas $(\cdot)^{H}$ denotes conjugate transpose (Hermitian). Additionally, I stands for the identity matrix. Finally, $\mathbb{E}[X]$ represents the expected value of a random variable $X$.

\section{SYSTEM MODEL AND PROBLEM STATEMENT}

In this section, we describe the HetNet that we have considered for our study. Such network is comprised of two tiers, namely, a set of $G_{m}$ macrocells, and a set of $G_{p}$ small cells located within the coverage area of the different macrocells, being $G=G_{m}+G_{p}$ the total number of cells. Hereinafter, and without loss of generality, we will refer to the latter as picocells; nevertheless, the results obtained in this work can be applied to a wider range of cell categories. Furthermore, the base stations (BS) corresponding to the macrocells have $N_{B S}$ antennas and transmit power $P_{B S}$, whilst the access points (AP) associated to the different picocells are equipped with $N_{A P}$ antennas and have a transmit power level of $P_{A P}$. Analogously, user equipments (UE) in both macro and picocells have $N_{U E}$ antennas and transmit power $P_{U E}$. Each cell handles its own intra-cell interference internally, thus we can model the scenario under study as a MIMO interference channel where each cell has a single active user and the different interfering links represent intercell interference. This setting is depicted in Fig. 1, where blue arrows represent downlink transmission and green lines correspond to the uplink.

\section{A. Interference Alignment in Flexible Duplexing networks}

As shown in Fig. 1, we allow the different cells in our network to be in either uplink or downlink within the same time slot. Each uplink/downlink configuration out of the $2^{G}$ total combinations is associated to a different MIMO interference channel. In order to handle inter-cell interference, we apply interference alignment, hence satisfying the following conditions

$$
\begin{aligned}
\mathbf{u}_{g}^{H} \mathbf{H}_{g, \ell_{j}} \mathbf{v}_{\ell_{j}}=0, & \forall g, j \neq g \\
\mathbf{u}_{g}^{H} \mathbf{H}_{g, j} \mathbf{v}_{j}=0, & \forall g, j \neq g \\
\mathbf{u}_{\ell_{j}}^{H} \mathbf{H}_{\ell_{j}, k_{g}} \mathbf{v}_{k_{g}}=0, & \forall j, g \neq j \\
\mathbf{u}_{\ell_{j}}^{H} \mathbf{H}_{\ell_{j}, g} \mathbf{v}_{g}=0, & \forall j, g \neq j .
\end{aligned}
$$

Condition (1a) is intended to cancel the interference generated by every $\mathrm{UE} \ell_{j}$ in uplink mode at the input of a base station (access point) $g$ in an uplink cell, where $\mathbf{u}_{g} \in \mathbb{C}^{N_{B S} \times 1}$ $\left(\mathbf{u}_{g} \in \mathbb{C}^{N_{A P} \times 1}\right)$ is the decoder, $\mathbf{v}_{\ell_{j}} \in \mathbb{C}^{N_{U E} \times 1}$ is the precoding vector and $\mathbf{H}_{g, \ell_{j}} \in \mathbb{C}^{N_{B S} \times N_{U E}}\left(\mathbf{H}_{g, \ell_{j}} \in \mathbb{C}^{N_{A P} \times N_{U E}}\right)$ represents the channel matrix from user $\ell_{j}$ to base station (access point) $g$. Analogously, condition (1b) is associated to the interference from a base station or access point in downlink mode at the input of a base station (access point) in uplink. Conditions (1c) and (1d) take into account the interference at the input of a UE in downlink configuration, coming from both users in uplink and base stations or access points in downlink, respectively. The dimensions of the different elements in conditions (1b) to (1d) can be straightforwardly derived as in condition (1a).

Furthermore, we have to guarantee that the desired signals within each cell are preserved, by satisfying the following conditions:

$$
\begin{aligned}
\mathbf{u}_{g}^{H} \mathbf{H}_{g, k_{g}} \mathbf{v}_{k_{g}} & \neq 0, \quad \forall g \\
\mathbf{u}_{\ell_{j}}^{H} \mathbf{H}_{\ell_{j}, j} \mathbf{v}_{j} & \neq 0, \quad \forall j .
\end{aligned}
$$

Note that conditions (2a) and (2b) refer to the rank preservation for both uplink and downlink cells, respectively.

One of the requirements for IA to be applied is the availability of channel state information (CSI). In our model, we consider an imperfect channel estimate defined as

$$
\hat{\mathbf{H}}_{g j}=\mathbf{H}_{g j}+\mathcal{E}_{g j} \quad \forall g, j,
$$

where $\mathcal{E}_{g j} \sim \mathcal{C N}(0, \eta \mathbf{I})$ with $\eta=\beta\left(\frac{1}{\sigma_{g}^{2}}\right)^{-\gamma}$ and $\sigma_{g}^{2}$ representing additive white Gaussian noise (AWGN) variance. Regarding parameter $\gamma$, we consider the hybrid model in [15]:

- For low-mid signal-to-noise ratio (SNR), $\gamma=1$ represents a scenario where the channel estimation impairments are dominated by noise.

- For high SNR, $\gamma=0$, we consider that the channel estimate errors are mainly due to quantization impairments, and hence their value remains constant with respect to noise variances.

The parameter $\beta$ is a control variable modeling the impact of SNR on channel estimation errors. 


\section{B. Problem statement}

In this context, our goal is to determine the uplink/downlink combination that maximizes the total downlink rate of the HetNet over 2 time slots, establishing an downlink-to-uplink time ratio of $1: 1$, i.e., all cells switch transmit direction in consecutive slots ${ }^{1}$. For this purpose, we propose the following discrete optimization problem:

$$
\begin{aligned}
\mathcal{P}_{1}: & \underset{\left\{i_{g}\right\}_{\forall g}}{\operatorname{maximize}} \sum_{g=1}^{G} R_{g}^{D} \\
& \text { subject to } i_{g}= \begin{cases}0 & \text { if cell } g \text { is in uplink } \\
1 & \text { if cell } g \text { is in downlink }\end{cases}
\end{aligned}
$$

where $R_{g}^{D}$ is the downlink rate for cell $g$ over the 2 considered time slots, i.e.,

$$
R_{g}^{D}=\log \left(1+\operatorname{SINR}_{g}^{D}\right) .
$$

The signal-to-interference-plus-noise-ratio (SINR) for a given UE in downlink, $\operatorname{SINR}_{g}^{D}$, is highly dependent on the transmission strategy implemented by the users in the network. Further, regardless of the transmission technique, the interference generated by the whole set of interfering cells is strongly related to the UL/DL configuration at any given time slot. For this reason, we include the control variables $i_{g}$ in the optimization problem, in such a way that the transmission direction of every cell is taken into account when the SINR is considered. Further details about the impact of variables $i_{g}$ on the SINR for all users are provided in Section III-B.

Although $\mathcal{P}_{1}$ is a general problem that encompasses any interference management technique, in the next section we explain in detail how the UL/DL configuration of these flexible duplexing scenarios can be optimized when IA is implemented.

\section{StATISTICAL ANALYSIS OF IA WITH IMPERFECT CSI}

As explained in Section II-B, a $G$-cell multi-tier network gives rise to $2^{G}$ uplink/downlink combinations, which are reduced to $2^{G-1}$ over 2 time slots due to the duality of the network in terms of rate. Therefore, evaluating the rate performance of every single setting is computationally demanding, especially due to the calculation of a different set of IA precoding and decoding vectors for each uplink/downlink setting. In order to reduce the complexity of determining the best combination, we have adapted the statistical characterization of IA presented in [16] to take into account the interference leakage due to the imperfect channel estimation.

\section{A. Interference leakage characterization}

In this section, we explain how the inter-cell interference leakage is modeled, following the same line as in [15]. From (3), with $\mathbf{H}_{g j}$ and $\mathcal{E}_{g j}$ being independent Gaussian variables, $\hat{\mathbf{H}}_{g j}$ and $\mathbf{H}_{g j}$ are jointly Gaussian. $\mathbf{H}_{g j}$ is Gaussian distributed

\footnotetext{
${ }^{1}$ For the sake of simplicity, we have considered a ratio 1:1, although more general settings, e.g. 4:1, can be found in practice.
}

with mean $\frac{\hat{\mathbf{H}}_{g j}}{1+\eta}$ and statistically independent elements of variance $\frac{\eta}{1+\eta}$. This allows us to rewrite the actual channel as

$$
\mathbf{H}_{g j}=\frac{1}{1+\eta} \hat{\mathbf{H}}_{g j}+\boldsymbol{\Upsilon}_{g j}
$$

where $\boldsymbol{\Upsilon}_{g j}$ is independent of $\hat{\mathbf{H}}_{g j}$, with distribution

$$
\Upsilon_{g j} \sim \mathcal{C N}\left(0, \frac{\eta}{1+\eta} \mathbf{I}\right)
$$

From (5) and the IA conditions in (1), we can state that the interference leakage at the input of receiver $g$ is given by

$$
\mathrm{IL}_{g}=\sum_{j \neq g} P_{j}\left(\frac{d_{g j}}{d_{0}}\right)^{-\alpha}\left|\hat{\mathbf{u}}_{g}^{H} \boldsymbol{\Upsilon}_{g j} \hat{\mathbf{v}}_{j}\right|^{2}
$$

where $\hat{\mathbf{u}}_{g}$ and $\hat{\mathbf{v}}_{j}$ are the decoders and precoders calculated from channel estimate $\hat{\mathbf{H}}$, i.e., $\left|\hat{\mathbf{u}}_{g}^{H} \hat{\mathbf{H}}_{g j} \hat{\mathbf{v}}_{j}\right|^{2}=0 . P_{j}$ is the transmit power for cell $j$, or equivalently, $P_{U E}$ in uplink cells and $P_{B S}\left(P_{A P}\right)$ in downlink cells. Additionally, $\left(\frac{d_{g j}}{d_{0}}\right)^{-\alpha}$ represents the normalized distance from transmitter $j$ to receiver $g$ with a path loss exponent $\alpha$.

Now, in order to obtain an expression characterizing the SINR for user $g, \mathrm{SINR}_{g}$, we need to obtain the interference leakage variance.

Lemma 1: $\mathbb{E}\left[\left|\hat{\mathbf{u}}_{g}^{H} \boldsymbol{\Upsilon}_{g j} \hat{\mathbf{v}}_{j}\right|^{2}\right]=\frac{\eta}{1+\eta}$

Proof: $\hat{\mathbf{H}}_{g j}$ and $\boldsymbol{\Upsilon}_{g j}$ are independent, and therefore $\hat{\mathbf{u}}_{g}$ and $\hat{\mathbf{v}}_{j}$ (calculated from $\hat{\mathbf{H}}_{g j}$ ) are independent of $\boldsymbol{\Upsilon}_{g j}$. Also, $\boldsymbol{\Upsilon}_{g j}$ is Gaussian distributed and bi-unitary invariant, thus the terms $\hat{\mathbf{u}}_{g}^{H} \boldsymbol{\Upsilon}_{g j} \hat{\mathbf{v}}_{j}$ are Gaussian random variables with zero mean and variance $\frac{\eta}{1+\eta}$. Since these terms are zero-mean:

$$
\begin{aligned}
\sigma_{I L_{g j}}^{2} & =P_{j}\left(\frac{d_{g j}}{d_{0}}\right)^{-\alpha} \mathbb{E}\left[\left|\hat{\mathbf{u}}_{g}^{H} \boldsymbol{\Upsilon}_{g j} \hat{\mathbf{v}}_{j}\right|^{2}\right] \\
& =P_{j}\left(\frac{d_{g j}}{d_{0}}\right)^{-\alpha} \frac{\eta}{1+\eta} \quad \forall g, j \neq g
\end{aligned}
$$

\section{B. Average rate performance}

Incorporating (7) to the statistical model in [16], the SINR for UE in cell $g$ is given by $\operatorname{SINR}_{g}=\rho_{g} X$, where $\rho_{g}$ is a deterministic constant given by

$$
\rho_{g}=\frac{\left\|\mathbf{H}_{k_{g} g}\right\|_{F}^{2} P_{g}\left(\frac{d_{k_{g} g}}{d_{0}}\right)^{-\alpha}}{\sigma_{k_{g}}^{2}+\sum_{j \neq g}\left[i_{j} P_{j}\left(\frac{d_{k_{g} j}}{d_{0}}\right)^{-\alpha}+\left(1-i_{j}\right) P_{\ell_{j}}\left(\frac{d_{k_{g} \ell_{j}}}{d_{0}}\right)^{-\alpha}\right] \frac{\eta}{1+\eta}}
$$

and $X \sim \operatorname{Beta}\left(1, N_{B S} N_{U E}-1\right)$ is a Beta distributed random variable ( $X \sim \operatorname{Beta}\left(1, N_{A P} N_{U E}-1\right)$ for picocells).

As proven in [16], since $\mathrm{SINR}_{g}$ follows a scaled Beta distribution, the average rate achievable by user equipment $k_{g}$ when a random IA solution from the set $\mathcal{S}$ is used is given by 


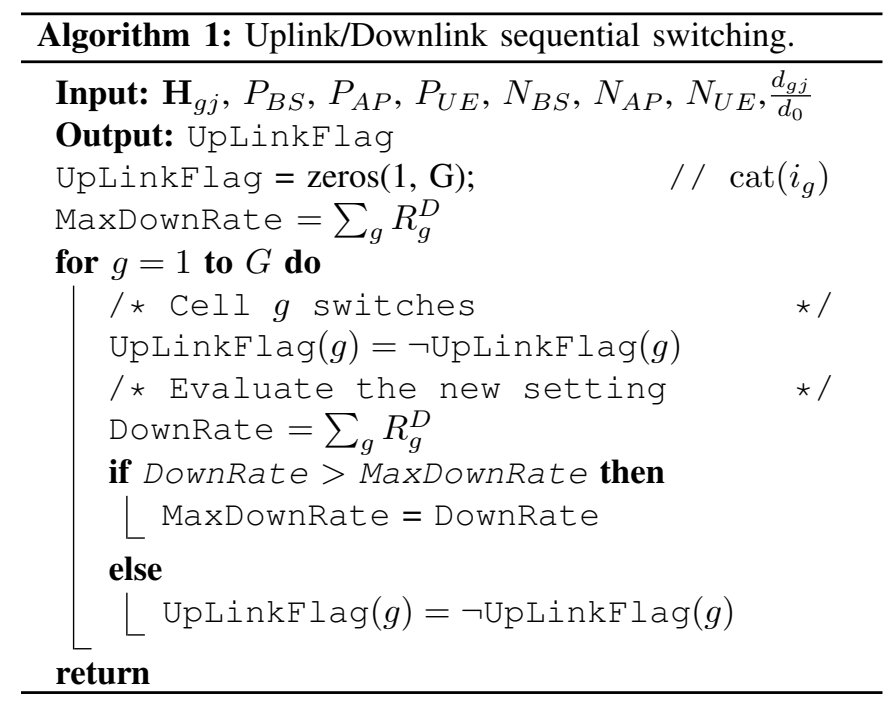

$$
\begin{aligned}
\mathbb{E}_{\mathcal{S}}\left[C_{g}\right] & =\mathbb{E}_{\mathcal{S}}\left[\log \left(1+\operatorname{SINR}_{g}\right)\right] \\
& =\left(\frac{\log (e) \rho_{g}}{N N_{U E}}\right){ }_{3} F_{2}\left(1,1,2 ; 2, N N_{U E}+1 ;-\rho_{g}\right)
\end{aligned}
$$

where ${ }_{3} F_{2}\left(a_{1}, a_{2}, a_{3} ; b_{1}, b_{2} ; x\right)$ denotes the generalized hypergeometric function. Notice again that analogous expressions are associated to the $N=N_{A P}$ antennas for access points in the picocells and $N=N_{B S}$ in the macrocells.

\section{Hierarchical uplink/downlink switching}

In order to reduce the computational cost of evaluating all the possible uplink/downlink combinations, we propose two different criteria to evaluate a subset of the total number of configurations in a given HetNet. For both of them, the main idea consists in starting with the given network in conventional TDD mode ${ }^{2}$. Then, a cell switches transmit direction with respect to the rest of cells, and the total downlink rate in the network is evaluated over 2 time slots so that all cells have transmitted in both directions at least once. If the downlink rate has been improved, the cell keeps the direction change, whereas if there is no improvement, the cell takes back to the previous state. The same procedure is repeated sequentially until every cell has evaluated the potential of the transmit direction switching. The switching sequence is described in Alg. 1.

The difference between the two proposed strategies originates from the hierarchical structure of the 2-tier HetNet under study. On the one hand, we can carry out the switching sequence in Alg. 1 starting with the macrocells (HS-Macro), and then continue with the picocells once macrocells have determined their best configuration. Nevertheless, as numerically observed by means of Monte Carlo simulations, picocells usually obtain the most significant benefit from flexible

\footnotetext{
${ }^{2}$ Conventional TDD is actually a particular case out of the total $2^{G-1}$ uplink/downlink settings in the HetNet
}

duplexing in terms of per-cell rate. Therefore, we also consider performing the sequence in Alg. 1 starting with the picocells first (HS-P i CO). Both approaches are compared to the optimal solution obtained by exhaustive search in Section IV.

\section{Simulation Results}

In this section we evaluate the benefits of flexible duplexing in terms of downlink rate for the 2-tier HetNets with imperfect CSI described in Section II. For this purpose, we consider two case studies:

- First, we analyze the rate improvements of flexible duplexing for a specific scenario which has been generated in order to observe how the different uplink/downlink settings affect the network behaviour.

- Afterwards, we have generated 100 independent spatial distributions for the nodes in the network, being the base stations at a deterministic location, whereas picocell access points and users of both tiers are distributed following a Poisson Point Process (PPP) around each BS location within its coverage area.

Throughout all our simulations, we take $\beta=0.1$, and we assume a channel quantization of 10 bits per MIMO channel matrix. This leads to an SNR threshold of $5 \mathrm{~dB}$ as calculated with the expressions in [17], so that $\gamma=1$ if SNR $<5 \mathrm{~dB}$, and $\gamma=0$ otherwise. Finally, notice that all the scenarios selected for evaluation satisfy the IA feasibility conditions in (1), (2).

We compare the results obtained by IA to those provided by the well-known minimum mean square error (MMSE) receiver. In this case, dominant eigenmode transmission (DET) is implemented at the transmitter side. Therefore, the MMSE filter at a given receiver $g, \mathbf{u}_{g}^{M M S E}$ is calculated as

$\mathbf{u}_{g}^{M M S E}=\mathbf{w}_{g}^{H} \hat{\mathbf{H}}_{g g}^{H}\left(\hat{\mathbf{H}}_{g g} \mathbf{w}_{g} P_{g}\left(\frac{d_{g g}}{d_{0}}\right)^{-\alpha} \mathbf{w}_{g}^{H} \hat{\mathbf{H}}_{g g}^{H}+\mathbf{R}_{g}+\sigma_{g}^{2} \mathbf{I}\right)^{-1}$

where $\mathbf{w}_{g} \in \mathbb{C}^{N \times 1}$ is the principal eigenvector of channel estimate $\hat{\mathbf{H}}_{g g}$, and $\mathbf{R}_{g}=\sum_{j \neq g}^{G} \hat{\mathbf{H}}_{g j} \mathbf{w}_{j} P_{j}\left(\frac{d_{g j}}{d_{0}}\right)^{-\alpha} \mathbf{w}_{j}^{H} \hat{\mathbf{H}}_{g j}^{H}$ is the covariance matrix of the inter-cell interference.

\section{A. Maximum downlink rate by exhaustive search}

In this case, we consider a network with $G_{m}=2$ macrocells and $G_{p}=3$ picocells. All nodes in the network are equipped with $N_{B S}=N_{A P}=N_{U E}=3$ antennas. Although power levels in local area network (LAN) standards are usually lower, we take into account the worst case scenario with the maximum transmit power for the UE in long-term evolution (LTE) communications, i.e, $P_{U E}=24 \mathrm{dBm}$ for all user equipments. Naturally, base stations and access points have a higher transmitted power, thus we set $P_{B S}=34 \mathrm{dBm}$ and $P_{A P}=31 \mathrm{dBm}$. The spatial distribution of the cells in terms of normalized distance is displayed in Fig. 2.

For the scenario in Fig. 2, the rate performance achieved by conventional TDD and the best flexible duplexing configuration have been evaluated over 100 independent channel realizations. 


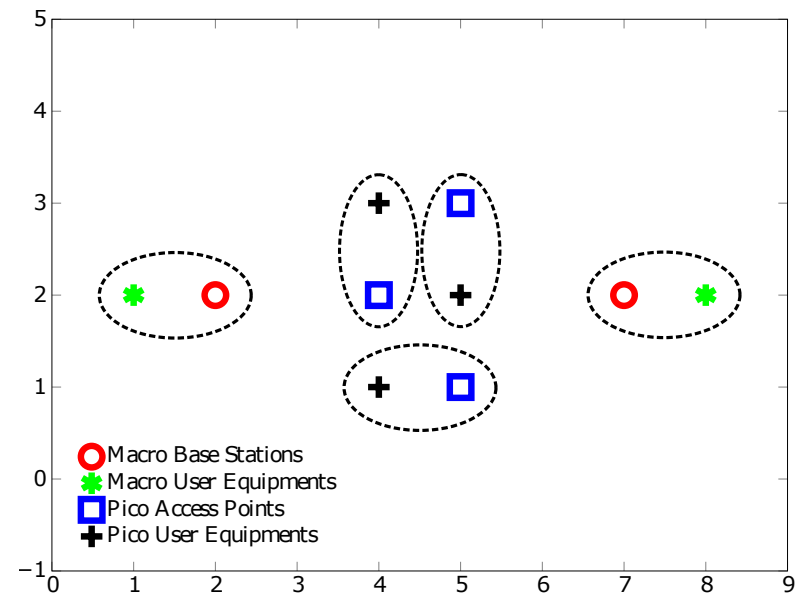

Fig. 2. Normalized locations of the nodes in the network (Scenario 1).

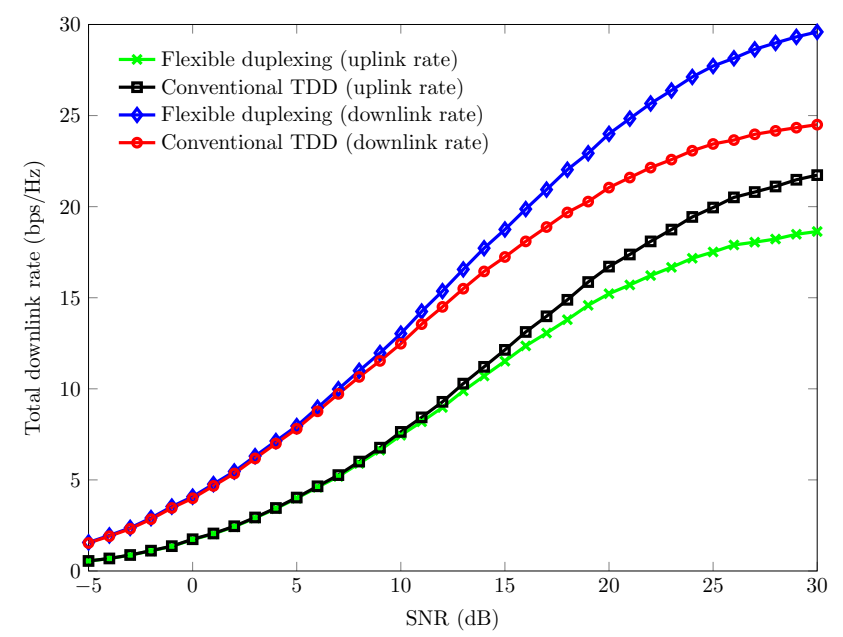

Fig. 3. Average rate over 100 independent channel realizations, obtained by conventional TDD and the UL/DL that maximizes donwlink rate.

The average downlink rates obtained by both approaches is shown for comparison in Fig. 3.

In this case, the optimal downlink rate is achieved when both macrocells are transmitting in the same mode as the upper-left picocell, whilst the remaining picocells are reversed. From the schematic representation in Fig. 2, it can be noticed that all picocells are located in the coverage edge of both macro BS, as well as significantly close to each other. Hence, when conventional TDD is applied, the inter-cell interference level generated at the input of the picocells users is considerably higher than that of the optimal flexible duplexing mode.

As observed in Fig. 3, the best flexible duplexing combination outperforms conventional TDD by approximately $25 \%$ in terms of downlink rate performance. Although this improvement comes at the price of a lower uplink rate, the uplink performance difference is still below $15 \%$ and thus the selected uplink/downlink combination provides the best overall performance as well.

Once we have studied the potential benefits of flexible duplexing over the conventional approach, we address the

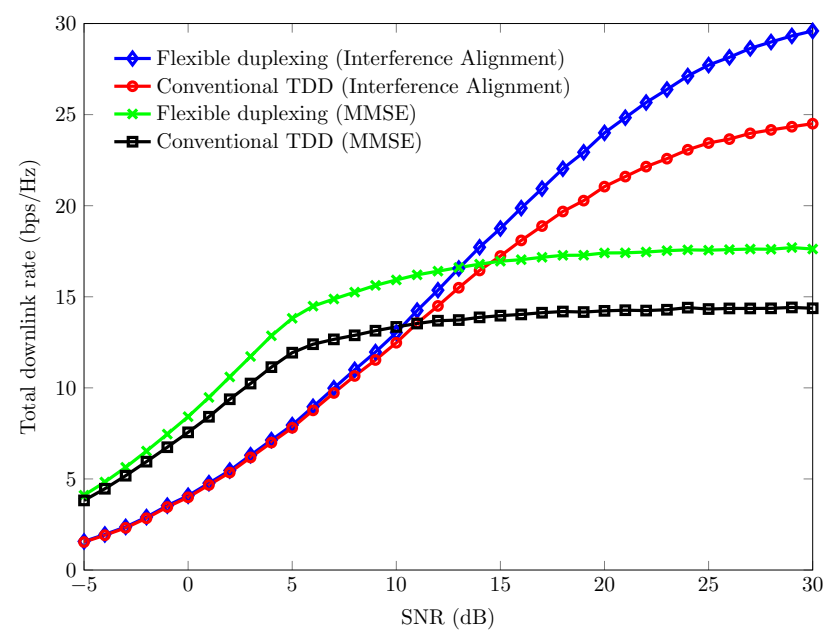

Fig. 4. Average downlink rate over 100 independent channel realizations, obtained by MMSE and IA.

comparison of the IA strategy with the well-known MMSE technique for the scenario depicted in Fig. 2. Again, we intend to find the UL/DL combination that maximizes total downlink rate over the 2 considered time slots, by means of exhaustive search. Figure 4 shows the total downlink rates obtained by both IA and MMSE. The comparison among conventional TDD and flexible duplexing is included as well for both schemes, in such a way that we can study the impact of every transmission technique on the potential benefits that UL/DL combining provides over standard TDD.

As expected, IA outperforms the MMSE approach with both conventional and Flexible TDD in the mid to high SNR regime. Notice that, for low SNR values, MMSE closes the gap with respect to IA. This is due to the strong relationship between the channel estimation impairments and the interference leakage level. More specifically, as detailed in Sections II-A and III-A, the channel estimation errors are modeled as a function of the SNR. Therefore, as the SNR decreases, the interference leakage remaining after applying the IA beamformers and filters is more significant, hence penalizing the performance.

Regarding the potential benefits that each transmission method can achieve by applying flexible duplexing, it can be observed in Fig. 4 that the improvement is similar for both techniques in absolute values. However, the impact of UL/DL optimization is higher for MMSE when compared to the performance of standard duplexing. It can be intuitively established that, since the residual interference after decoding is more significant in the case of MMSE, this strategy will be more benefited when finding the UL/DL combination that minimizes the inter-cell interference at the receivers.

\section{B. Hierarchical approaches for PPP distributed deployments}

As mentioned in Section III-C, the computational cost of evaluating all the possible flexible duplexing combinations by exhaustive search is significantly high, even when statistical rate expressions allow to avoid the calculation of IA solutions for every single uplink/downlink setting. In this section, we 


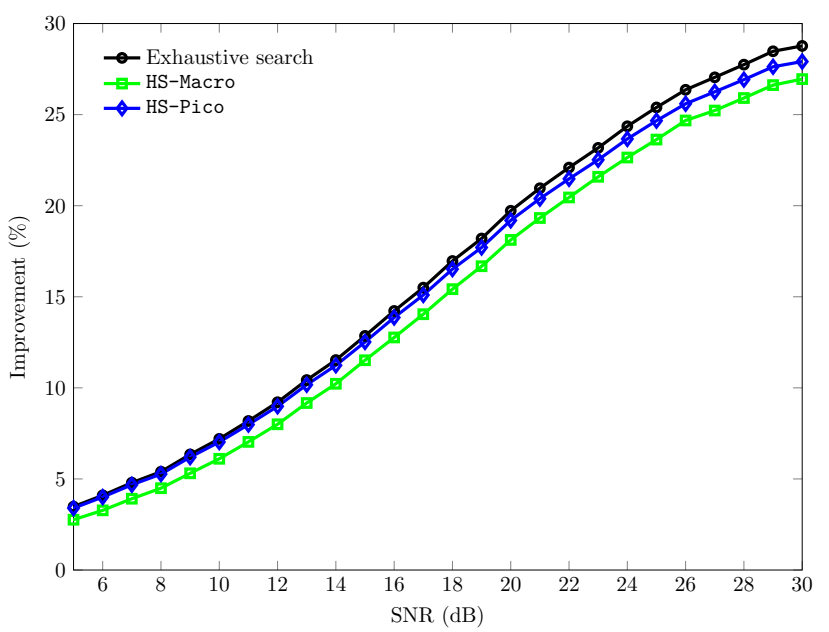

Fig. 5. Downlink rate improvement (\%) of flexible duplexing over conventional TDD.

evaluate the two proposed hierarchical heuristic approaches introduced in Algorithm 1, and we compare them to the optimal solution. For that purpose, we generate 100 independent PPP spatial distributions of a 2-tier HetNet with 2 macrocells and 4 picocells, each one associated to an independent channel realization. BS and AP are equipped with $N_{B S}=N_{U E}=5$ antennas, whereas every UE has $N_{U E}=2$ antennas. The transmit power levels remain the same as in Section IV-A.

Figure 5 shows the downlink rate improvement of the three strategies with respect to conventional TDD, averaged over the 100 independent PPP realizations. From the black curve, representing the improvement attained with exhaustive search among all the possible combinations, we can establish that flexible duplexing outperforms conventional transmission by up to almost $30 \%$ at high-SNR regimes. As expected, the HS-Pico approach slightly outperforms its analogous HS-Macro. Notice that, as discussed in Section IV-A, the picocells of the network get the most benefit of flexible duplexing when compared to the macro tier. Finally, it can be observed that both hierarchical approaches provide rates significantly close to the results obtained by means of exhaustive search, with the additional advantage of their reduced computational cost.

\section{CONCLusion}

In this work, we have addressed a rate analysis of 2-tier flexible duplexing MIMO HetNets where IA precoders and decoders are applied to cancel inter-cell interference. Since determining the optimal uplink/downlink configuration that maximizes the total downlink rate is a combinatorial problem, we have developed approaches to reduce the computational cost associated to the evaluation of each up/down setting. By means of Monte Carlo simulations, we have studied the performance of the proposed techniques. At low-mid SNR, the channel estimation errors are dominated by noise, and hence flexible duplexing has no noticeable impact on intercell interference variances. Nevertheless, we have shown that implementing flexible duplexing provides remarkable benefits when compared to conventional TDD transmissions, reaching improvements of almost $30 \%$ in high-SNR, interference limited regimes.

\section{ACKNOWLEDGMENT}

This work has been supported by the Ministerio de Economía, Industria y Competitividad (MINECO) of Spain under grant TEC2016-75067-C4-4-R (CARMEN), and FPI grant BES-2014-069786.

\section{REFERENCES}

[1] X. Chu, D. Lopez-Perez, Y. Yang, and F. Gunnarsson, Heterogeneous Cellular Networks. Cambridge, 2013.

[2] J. Hoydis, K. Hosseini, S. t. Brink, and M. Debbah, "Making smart use of excess antennas: Massive MIMO, Small Cells, and TDD," Bell Labs Technical Journal, vol. 18, no. 2, pp. 5-21, 2013. [Online]. Available: http://dx.doi.org/10.1002/bltj.21602

[3] M. Kountouris and N. Pappas, "HetNets and massive MIMO: Modeling, potential gains, and performance analysis," in 2013 IEEE-APS Topical Conference on Antennas and Propagation in Wireless Communications $(A P W C)$, Sep. 2013.

[4] L. Sanguinetti, A. L. Moustakas, and M. Debbah, "Interference management in 5G reverse TDD HetNets with wireless backhaul: A large system analysis," IEEE Journal on Selected Areas in Communications, vol. 33, no. 6, pp. 1187-1200, Mar. 2015.

[5] I. Randrianantenaina, H. Dahrouj, H. Elsawy, and M.-S. Alouini, "Interference management in Full-Duplex cellular networks with partial spectrum overlap," IEEE Access, vol. 5, pp. 7567-7583, Mar. 2017.

[6] S. Lembo, O. Tirkkonen, M. Goldhamer, and A. Kliks, "Coexistence of FDD flexible duplexing networks," in 2017 European Conference on Networks and Communications (EuCNC), Jun. 2017.

[7] J. Kerttula, A. Marttinen, K. Ruttik, R. Jäntti, and M. N. Alam, "Dynamic TDD in LTE small cells," EURASIP Journal on Wireless Communications and Networking, vol. 2016, no. 1, Aug. 2016.

[8] S. Lagen, A. Agustin, and J. Vidal, "Joint user scheduling, precoder design, and transmit direction selection in MIMO TDD small cell networks," IEEE Transactions on Wireless Communications, vol. 16, no. 4, pp. 2434-2449, Mar. 2017.

[9] K. Lee, Y. Park, M. Na, H. Wang, and D. Hong, "Aligned reverse frame structure for interference mitigation in dynamic TDD systems," IEEE Transactions on Wireless Communications, vol. 16, no. 10, pp. 6967-6978, Oct. 2017.

[10] S.-W. Jeon and C. Suh, "Degrees of Freedom of UplinkDownlink Multiantenna Cellular Networks," 2014. [Online]. Available: http://arxiv.org/abs/1404.6012

[11] K. Kim, S.-W. Jeon, J. Yang, and D. K. Kim, "The feasibility of interference alignment for MIMO interfering broadcastmultiple-access channels," IEEE Transactions on Wireless Communications, vol. 16, no. 7, pp. 4614-4625, Jul. 2017.

[12] J. Fanjul, Oscar González, I. Santamaria, and C. Beltrán, "Homotopy continuation for spatial interference alignment in arbitrary MIMO X networks," IEEE Transactions on Signal Processing, vol. 65, no. 7, pp. 1752-1764, Apr. 2017.

[13] J. Fanjul and I. Santamaria, "On the spatial degrees of freedom benefits of reverse TDD in multicell MIMO networks," in 2016 24th European Signal Processing Conference (EUSIPCO), Aug. 2016.

[14] C. M. Yetis, J. Fanjul, J. A. Garcia-Naya, N. N. Moghadam, and H. Farhadi, "Interference alignment testbeds," IEEE Communications Magazine, vol. 55, no. 10, pp. 120-126, Oct. 2017.

[15] P. Aquilina and T. Ratnarajah, "Performance Analysis of IA Techniques in the MIMO IBC With Imperfect CSI," IEEE Transactions on Communications, vol. 63, no. 4, pp. 1259-1270, Apr. 2015.

[16] I. Santamaria and J. Fanjul, "Statistical analysis of single-beam interference alignment schemes," in 2016 IEEE 17th International Workshop on Signal Processing Advances in Wireless Communications (SPAWC), Jul. 2016.

[17] S. Shrestha, G. Fang, E. Dutkiewicz, and X. Huang, "Effect of CSI quantization on the average rate in MU-MIMO WLANs," in 2016 13th IEEE Annual Consumer Communications and Networking Conference (CCNC), Jan. 2016. 